\title{
High Strength Steel (HSS) characterization for numerical simulation of defence applications: from data collection to triaxiality model validation
}

\author{
Jerome Mespoulet ${ }^{1 *}$, Hakim Habdulhamid ${ }^{1}$, and Paul Deconinck ${ }^{1}$ \\ ${ }^{1}$ Shock Physics Department, THIOT INGENIERIE, 830 Route Nationale, France
}

\begin{abstract}
This paper details the methodology developed to fit a material strength/failure model with mesh sensitivity, strain rate influence and triaxiality effect on plastic failure for a blast/ballistic configuration for a HSS plate. Data collection in different loading conditions in quasi-static and dynamic regimes has been done, following a given tests matrix based on the customer phenomenological analysis. A complete set of parameters has been find by numerically reproduce the tests and find the best compromise to fit all the tests performed with a unique set. Tests on large square clamped plates have been performed with three nose shapes (flat, hemispheric and conical) to validate the set of parameters. One case with perforation and another at failure initiation have been done for each geometry. Numerical works using the final set of parameters have shown very good agreement in the various impact conditions.
\end{abstract}

\section{Introduction}

High Strength Streel (HSS) and Advanced High Strength Steel (AHSS) are commonly used as structural components in construction and transport applications. S890QL grade for mobile crane industry and lifting components or HY-100 for submarine hull are some examples where combination of high strength and ductility is a mandatory.

Systems and components in the Defence field are others candidates who needs to take benefit of the HSS family of materials due to threat they have to face off. They are selected as structure materials to maintain stiffness of vehicles and/or as shielding to protect carrier from bullets, bomb fragments, buried charge, Improvised Explosive Device (IED) or unconventional heavy projectiles.

Selection of the appropriate strength \& rupture model(s), material characterization and implementation in dynamic software for simulation of impact or blast events need to follow some rules to allow good prediction and ensure geometry optimization for designers.

*Corresponding author: mespoulet@,thiot-ingenierie.com 


\section{Context}

Even if aluminum grades usage in tactical vehicles increase continuously for armor, hull and frames, Rolled Homogeneous Armor (RHA), HSS and AHSS as structural components are still inevitable to fulfill all requirements surrender in operation [1]. Continuous needs coming from automotive sector manage to save weight by increasing Ultimate Tensile Strength (UTS) at the expense of Total Elongation (TE). This is done over the last five decades by mastering the microstructure from single phase High-Strength Low-Alloy Steel (HSLA), to Dual Phase (DP), Transformation-Induced Plasticity (TRIP), TWinning Induced-Plasticity (TWIP) or, for example, Martensitic steels (SMART). Various generation of AHSS have been developed to increase the UTS * TE constant has shown by Fig. 1-a and the amount of AHSS becomes higher and higher since the 90s' in automotive in North America (Fig. 1-b).
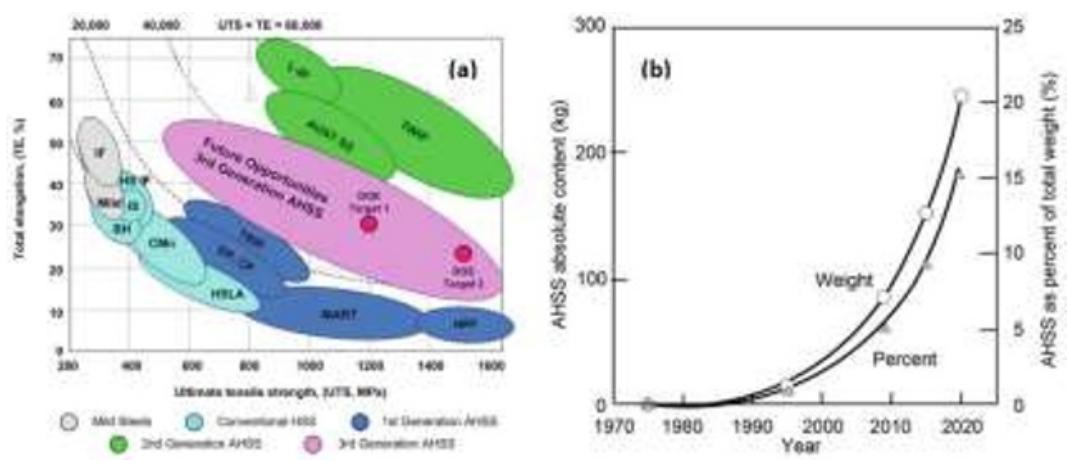

Fig. 1. Total Elongation versus Ultimate Tensile Strength classification of HSS and AHSS (a) and historical usage evolution of AHSS in automotive in North America (b) [2]

This quest to access simultaneously high strength and elongation for civilian applications is also a goal for military purposes. In this field, the aim is to protect personal carrier crews [3-5] by defeating and reducing damage effects generated by blast/impact of conventional ammunitions or IEDs. The threat can either be a fragments cloud or an underbelly buried charge that manufacturer can evaluate experimentally or by dynamic numerical analysis (Fig.2).
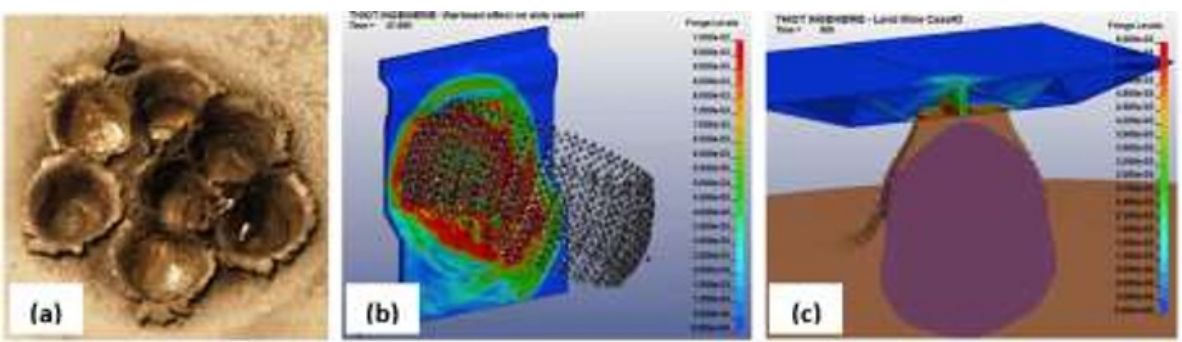

Fig. 2. Synchronized impact of seven steel fragments (a) ; Warhead fragments impact on a tactical vehicle side panel (b) and underbelly buried charge explosion determination (c)

\section{Material model description}

This study aims at calibrating a material model in LSDYNA ${ }^{\mathrm{TM}}$ in order to improve the predictive performances of numerical simulations of blast and impact loading on HSS structures. The MAT_224 material model in LSDYNA ${ }^{\mathrm{TM}}$ was imposed by the final customer. 
This model is a tabulated version of the Johnson-Cook model with failure strain dependency. It has originally been developed to reproduce Ti-6Al-4V behaviour under a common project between George Mason University, Ohio State University, NASA John H. Glenn Research Center and the Federal Aviation Administration Aircraft Failure Prevention Program [7]. The constitutive law follows Von Mises type yield surface is isotropic elasto-thermo-visco-plastic and the yield surface is defined by two series of curves with stress versus strain: the first at different temperature level and second set at different strain rate.

The evolution of the strain failure is governed by another 3D surface that depends on the triaxiality and the Lode Angle. This evolution is after scaled using a multiplicative formulation of individual influence of temperature, strain rate and element size.

\section{Study methodology}

The presented methodology is built on our expertise in shock physics and material dynamics, which is based on a continuous dialogue between tests and simulations. This approach has been validated on various research projects related to material dynamic behaviour characterization. Taking advantages of both, means reducing research time and cost. On one hand, experimental tests help identify the most prominent phenomena to consider and provide data for model parameters. On the other hand, numerical simulations improve the comprehension of phenomena mechanisms especially in the case of dynamic events that may be too brief to be experimentally observed. Numerical simulation also helps in the design of test setups and conditions in order to reduce iterations. This project therefore used an iterative process between tests and numerical simulations with a Building Block Approach (BBA). BBA consists in analysing the material response from mesoscopic to structure scales. It was applied in this paper to calibrate a material model in LS-DYNA ${ }^{\mathrm{TM}}$ of a HSS material subjected to blast and impact loading.

A phenomenological analysis was first introduced (not detailed in this paper) in order to identify the important parameters to consider for material modelling with respect to the final application. It also helped defining the range of strain rate, stress triaxiality and Lode angle levels associated to the concerned threats. The BBA was then defined to find the best compromise between cost, project duration and precision of the material model calibration:

- Model calibration through several quasi-static and some dynamic tests at different temperatures and stress triaxiality levels,

- Model adjustment through dynamic tensile and compression test (at different strain rates and temperatures) and associated numerical simulations,

- Validation tests through high energy impact experiments and final simulation to evaluate the prediction capability of the calibrated model.

These three steps are presented in the following three chapters.

\section{Model calibration}

The MAT_224 material model being a multiplicative relation of each contribution (stress triaxiality, strain rate and temperature), the effect of each contribution on both the stress and the failure strain were investigated separately with the following tests:

- Quasi-static tensile tests on smooth specimens: allowing to define the behaviour of the material up to failure in static condition,

- Quasi-static tensile tests at various temperature: allowing to define the behaviour of the material up to failure as a function of temperature,

- Quasi-static tensile (on specific specimen geometries) and torsional tests: to define the failure strain evolution as a function of stress triaxiality, 
- Dynamic tensile tests (on Split Hopkinson Tensile Bars) on smooth specimens: allowing the identification of the strain rate sensitivity of stress and failure strain.

At the end, the MAT_224 material model was calibrated and numerical simulation of most of the tests were performed. The comparison of tests results and numerical simulations for two configuration is illustrated in Fig. 3. A good correlation is obtained between the stress vs strain response and failure strain.
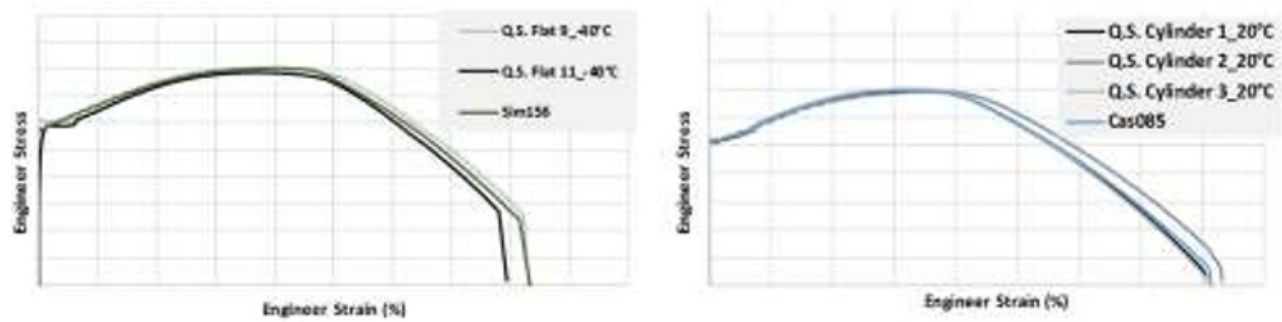

Fig. 3. Quasi-static tests results on different sample geometries and temperatures. Comparison with numerical simulations with the calibrated material model.

\section{Model adjustment}

The following step consists in evaluating the material model parameters previously identified on more complex test configurations. Most of these tests were performed under dynamic loading on Split Hopkinson Tensile and Torsional bars (Fig. 4.). This allowed to mix each contribution (strain rate, temperature and stress triaxiality) on the same experiment.

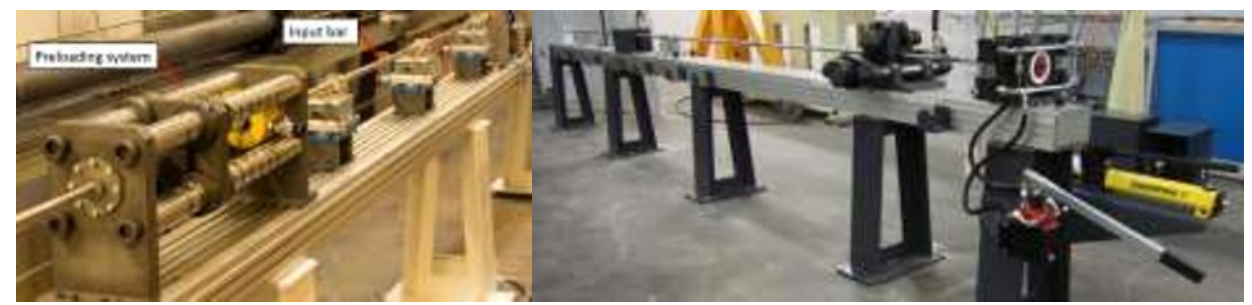

Fig. 4. Split Hopkinson Tensile (left) and Torsional (right) Bars

The SHTB tests on flat specimens were observed using a Phantom V2012 high-speed camera. A 2D Digital Image Correlation (DIC) was also done on this configuration to evaluate the evolution of the strain field during the tests (see Fig. 5.). Dynamic tracking of some characteristic points have been selected on 2 d-axisymetric tensile samples (as illustrated in Fig. 6.).

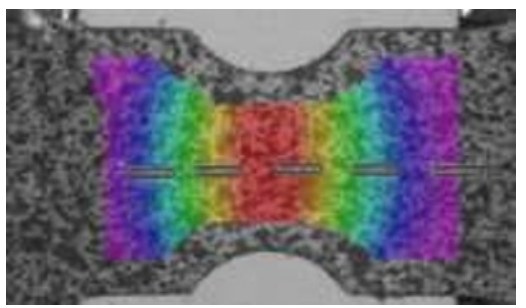

Fig. 5. Example of 2D-DIC analysis

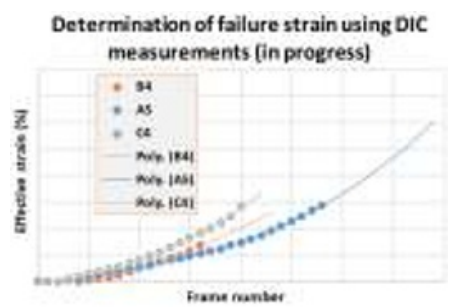

Fig. 6. Example of dynamic tracking analysis 


\section{DYMAT 2021}

The material model parameters were then adjusted and final simulations of most of the tests were performed. Fig. 7. presents a comparison between test and numerical results. A good correlation was find, highlighting the ability of the material model to faithfully reproduce the behaviour up to failure in complex conditions.
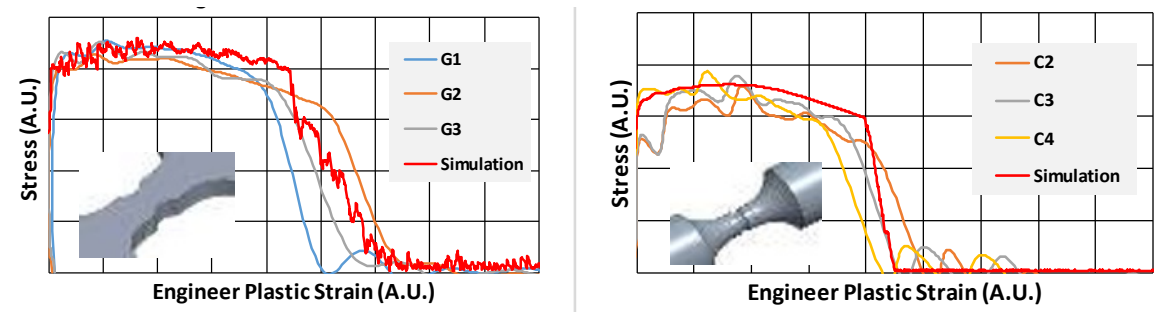

Fig. 7. Comparison between simulations and SHTB tests with various triaxiality levels

\section{Model evaluation}

The final step of the BBA is the evaluation of the predictiveness of the material model on representative test configuration, allowing to load the material in conditions close to the final application. The chosen configuration is an impact setup on clamp targets made of HSS material (Fig. 8.). Different nose shapes (flat, conical and hemispherical) were investigated in order to highlight various failure mode. For each projectile shape, two velocities were investigated to reach different failure level (failure initiation and total perforation).

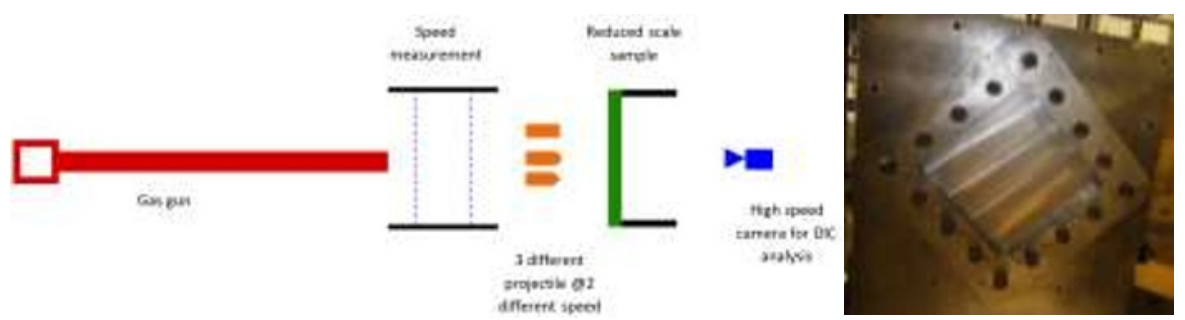

Fig. 8. Test configuration for the punch tests campaign

The velocity of the impactor was measured using an optical velocimeter before impacting the target. 2 high-speed cameras were positioned to observe the back face of the target during the impact test with 3D DIC analysis (Fig. 9).

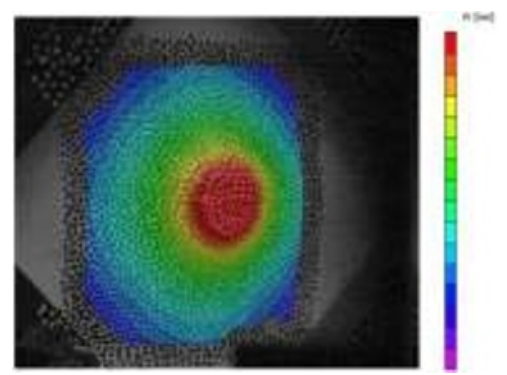

Fig. 9. Test configuration for the impact tests campaign

Finally, all 6 impact tests were simulated with the previously adjusted material model in LSDYNA $^{\text {TM }}$. Fig. 10. shows the correlation between one numerical simulation and the 
corresponding test. They showed very good agreement in terms of residual kinetic energy, z-displacement field and time for failure initiation.
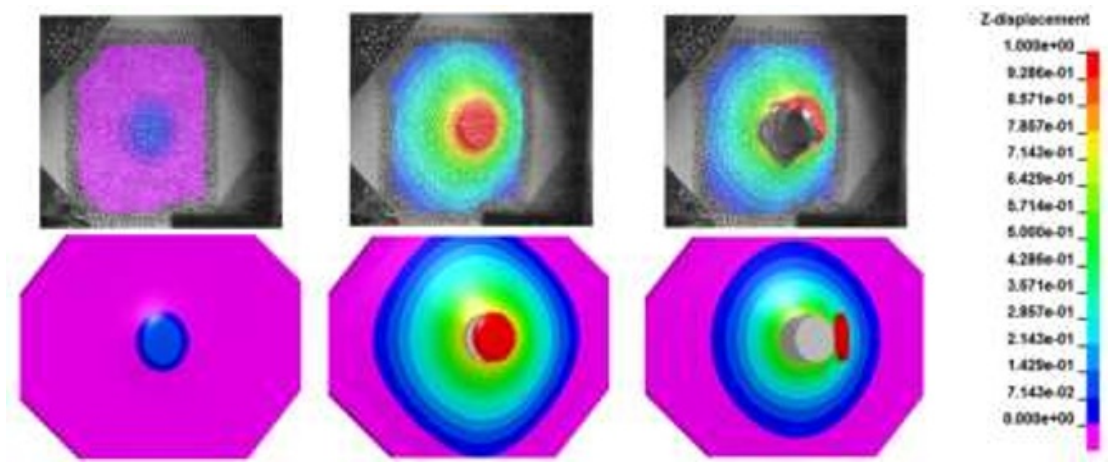

Fig. 10. Clamped plate perforation with flat nose projectile: comparison of Z-displacement of the back face (Arbitrary Unit, $0.4 \mathrm{~ms}$ interframe) between 3D-DIC analysis (top) and simulation (bottom)

\section{Conclusions}

This paper aimed at presenting a characterization methodology adapted to HSS subject to blast or impact loading. A MAT_224 material model in LSDYNA ${ }^{\mathrm{TM}}$ was calibrated through several steps starting from coupon testing to evaluate the individual effects of strain rate, temperature and stress triaxiality, up to scale 1 impact tests. At the end, the calibrated model is able to correctly predict the impact results. This methodology called BBA can be applied for any kind of material and has led to reliable results thanks to an iterative process between tests and numerical simulations.

\section{References}

1. T. Slezak, Employment of the new advanced structural materials in the military vehicles and heavy equipment, in Proceedings of the $1^{\text {st }}$ International Challenges to national Defence in Contemporary Geopolitical Situation, CNDCGS, 25-27 April 2018, Vilnius, Lithuania (2018)

2. M.Tisza, Development of Lightweight Steels for Automotive Applications in Engineering Steels and High Entropy-Alloys book, DOI: 10.5772/intechopen.91024, (2020)

3. K. Williams and F. Fillion-Gourdeau, Numerical simulation of Light Armoured Vehicle Occupant Vulnerability to Anti-Vehicle Mine Blast, in proceedings of the 7th International LS-DYNA Users Conference, Detroit, (2002)

4. Z. Hryciow and G. Slawinski, Investigation of armoured personnel carrier crew subjected to impact load, in proceedings of Computational Technologies in Engineering, TKI 2018, AIP Conference 2078, (2018)

5. G. Slawinski, P. Malesa and M. Swierczewski, Analysis Regarding the Risk of Injuries of Soldiers Inside a Vehicle during Accidents Caused by Improvised Explosive Devices, Appl. Sci., 9, p4077 2078, (2019)

6. S. Haight, L. Wang, P Du Bois, K. Carney, C-D Kan, Development of Titanium Alloy Ti-6Al-4V Material model Using in LS-DYNA, Final report ${ }^{\circ}$ DOT/FAA/TC-15/23, May 2016, (2016) 